\title{
Funções e ordem da razão dos nutrientes no estabelecimento de normas DRIS em laranjeira 'Valência'(1)
}

\author{
Francisco de Assis Alves Mourão Filho(2), João Carlos Azevedo(2) e Josef Andreas Nick ${ }^{(3)}$
}

Resumo - O sistema integrado de diagnose e recomendação DRIS (Diagnosis and Recommendation Integrated System) tem sido uma boa alternativa para a avaliação do estado nutricional de diversas culturas. O objetivo deste trabalho foi calcular índices DRIS mediante o uso de três métodos (Beaufils, Elwali \& Gascho e Jones) de cálculo das funções das razões dos nutrientes e de dois critérios (Letzch e Nick) para a escolha da ordem da razão dos nutrientes, em pomares comerciais irrigados de laranjeira 'Valência'. Informações sobre a produtividade, porta-enxerto e teor de N, P, K, Ca, Mg, S, Fe, Mn, Cu, Zn e B em folhas de ramos não-frutíferos de cada talhão foram processadas nos anos de 1994 a 1998. O índice de balanço nutricional (IBN) foi calculado pela média da soma dos valores em módulo dos índices nutricionais e do índice de matéria seca. Os índices DRIS e IBN foram calculados e os valores de $\mathrm{r}^{2}$ (produtividade em função de IBN) foram obtidos para cada método. O método Jones para cálculo do DRIS apresentou melhor correlação com a produtividade, e o critério Nick mostrou-se mais eficiente na escolha da ordem da razão dos nutrientes. Populações específicas, com pequeno número de observações, padronizadas quanto ao porta-enxerto e referentes a um ou dois anos de amostragem foliar e produção foram bancos de dados eficientes para a obtenção das normas DRIS

Termos para indexação: Citrus sinensis, diagnose foliar, equilíbrio de nutrientes, estado nutricional, método de análise.

Functions and ratio order of the nutrients at the establishment of DRIS norms in 'Valencia' sweet orange

\begin{abstract}
Diagnosis and Recommendation Integrated System (DRIS) has been a useful tool for nutrient diagnosis of several crops. This research aimed to establish adequate norms for DRIS through three methods (Beaufils, Elwali \& Gascho and Jones) to calculate ratio functions and two criteria (Letzsch and Nick) for choosing the ratio order of the nutrients for 'Valencia' sweet orange budded on three rootstocks, in Mogi Guaçú, SP, Brazil. Data referred to yield, rootstock and concentrations of N, $\mathrm{P}, \mathrm{K}, \mathrm{Ca}, \mathrm{Mg}, \mathrm{S}, \mathrm{Fe}, \mathrm{Mn}, \mathrm{Cu}, \mathrm{Zn}$ and $\mathrm{B}$ in leaves of non fruiting terminals, for each plot, were processed for 1994 through 1998. Nutritional Balance Index (NBI) was calculated by the average of the sum (disregarding the sign) of all nutritional index values and dry matter index. DRIS and NBI indexes were calculated and the $\mathrm{r}^{2}$ values (yield related to NBI) were obtained for each calculation method. DRIS calculation method of the ratio function, recommended by Jones, presented better correlation with yield. The criterion for choosing the ratio order of the nutrients recommended by Nick was more efficient. Specific populations, with reduced number of observations, standardized by rootstock and referred to one or two sampling years were more efficient data for calculation of DRIS norms.
\end{abstract}

Index terms: Citrus sinensis, foliar diagnosis, nutrient balance, nutritional status, analytical methods.

(1) Aceito para publicação em 21 de maio de 2001

(2) Escola Superior de Agricultura Luiz de Queiroz (Esalq), Dep. de Produção Vegetal, Caixa Postal 9, CEP 13418-900 Piracicaba, SP. Bolsista CNPq.

E-mail: famourao@esalq.usp.br

(3) Caixa Postal 0686, Guapirama, PR

E-mail: nick-cafe@uol.com.br

\section{Introdução}

Os métodos usuais para interpretação de análises químicas em folhas pressupõem a comparação das concentrações de nutrientes encontradas, com valores de referência (concentrações críticas ou faixas de suficiência). O Sistema Integrado de Diagnose 
e Recomendação (DRIS = Diagnosis and Recommendation Integrated System) é outra forma de interpretação dos resultados de análise foliar, e utiliza relações entre nutrientes, em vez da concentração absoluta e isolada de cada um deles. O método DRIS estabelece índices que significam, em uma escala numérica contínua, o efeito de cada nutriente no balanço nutricional da planta. Esses índices são expressos por valores positivos ou negativos. Valores negativos indicam que o referido nutriente se encontra deficiente, e valores positivos significam que o nutriente se encontra em excesso em relação aos demais. Quanto mais próximos de zero estiverem os índices de todos os nutrientes, mais próxima estará a planta de seu equilíbrio nutricional (Jones, 1981; Beverly, 1991).

As normas DRIS são obtidas sempre de uma população de alta produtividade, denominada população de referência, que é selecionada a partir de uma população maior dentro de um conjunto de dados também criteriosamente selecionado. Os bancos de dados para obtenção das normas podem ter tamanho variável em razão das premissas a serem adotadas no método, e devem ser uniformes quanto às características da cultura (Letzsch \& Sumner, 1984).

A população ou banco de dados escolhida para definição das normas deve ser subdividida em duas subpopulações ou categorias: uma, constituída de plantas normais, ou população de referência, com produtividade significativamente superior a um nível criteriosamente estabelecido; e outra, com plantas anormais, ou população não-referência, com produtividade menor que o estabelecido (Beaufils, 1973; Beverly, 1991).

Nos cálculos do método DRIS, apenas um tipo de expressão é utilizado para relacionar cada par de nutrientes. Entre os diversos critérios para selecionar a expressão mais adequada, um dos mais utilizados é o critério da maior relação de variâncias entre as populações de alta e baixa produtividade (Letzsch, 1985). Nick (1998) propôs o critério denominado "Valor r" para a escolha da ordem da razão dos nutrientes na aplicação do DRIS. Esse critério compreende o cálculo dos coeficientes de correlação (r) entre os valores da produtividade (variável resposta da planta) e a razão entre os pares de nutrientes, tanto na ordem direta como na ordem inversa. É escolhida a ordem da razão que resultar no mais alto valor absoluto do coeficiente de correlação.

Para calcular as funções das razões dos nutrientes, existem três métodos: a) o método original, proposto por Beaufils (1973); b) o método Beaufils (1973) modificado por Elwali \& Gascho (1984); c) o método Jones (1981). Embora esses métodos de cálculos de funções das razões dos nutrientes tenham sido avaliados em alguns trabalhos, o mais recomendável ainda não foi definido.

Para o somatório das funções envolvendo cada nutriente, existem dois métodos citados por Beverly (1991): o DRIS (Beaufils, 1973) e o M-DRIS (Hallmark et al., 1987). O método DRIS original utiliza-se apenas das funções das razões entre nutrientes. Já o método M-DRIS, variação e expansão do método DRIS, prevê a inclusão da matéria seca no cálculo dos índices. As expressões são idênticas às normalmente utilizadas, mas, neste caso, a matéria seca é tratada como um constituinte adicional e um índice é calculado para matéria seca, da mesma forma como para os demais constituintes vegetais.

Pesquisas realizadas nos Estados Unidos, África do Sul, Espanha e Venezuela relatam investigações sobre DRIS em citros (Wallace, 1990; Beverly, 1992; Woods \& Villiers, 1992; Cerda et al., 1995; Moreno et al., 1996; Rodriguez et al., 1997). No Brasil, Bataglia (1989) ressaltou que esse método pode ser uma alternativa para a diagnose nutricional de citros, mas que deve ser utilizado em conjunto com os demais critérios de diagnose. Creste (1996) demonstrou vantagem no uso do DRIS em relação ao método de faixas de suficiência, em limoeiro 'Siciliano', principalmente devido à sua capacidade de indicar os nutrientes deficientes ou em excesso, em sua ordem de importância. Santos (1997) obteve resultados em que o método DRIS foi superior ao critério de faixas de suficiência na detecção de plantas aquém do seu potencial de produtividade, em face de limitações nutricionais. Entre os três procedimentos de cálculo dos índices DRIS, o proposto por Jones (1981) foi o que apresentou mais vantagens.

O objetivo deste trabalho foi estabelecer normas DRIS por meio de três formas de cálculo das funções das razões dos nutrientes e de dois critérios para a escolha da ordem da razão dos nutrientes em poma- 
res comerciais irrigados de laranjeira 'Valência' sobre três porta-enxertos.

\section{Material e Métodos}

A coleta dos dados experimentais foi realizada conjuntamente com o Grupo Sete Lagoas Agrícola S/A (Fazenda Sete Lagoas), em Mogi Guaçú, SP. A localização geográfica da propriedade é definida pelas coordenadas geográficas $22^{\circ} 12^{\prime} 30^{\prime \prime}$ de latitude Sul, $47^{\circ} 12^{\prime} 0^{\prime \prime}$ de longitude Oeste, e altitude média de $620 \mathrm{~m}$. O solo predominante é classificado como Latossolo Vermelho-Amarelo distrófico amarelado, textura média.

A variedade-copa utilizada neste estudo foi a laranjeira 'Valência' (Citrus sinensis, L. Osbeck). Todos os talhões selecionados para o estudo foram irrigados, apresentando idade superior a seis anos e produtividade superior a $20 \mathrm{t} / \mathrm{ha}$. O banco de dados formado incluiu a discriminação do talhão também em função do porta-enxerto. Os talhões de laranjeira 'Valência' estavam sobre os porta-enxertos: limoeiro 'Cravo' (Citrus limonia L. Osbeck), laranjeira 'Caipira' (Citrus sinensis, L. Osbeck), Trifoliata (Poncirus trifoliata, Rafinesque), limoeiro 'Volkameriano' (Citrus volkameriana, Pasquale), citranges 'Troyer' e 'Carrizo' (Citrus sinensis $\mathrm{x}$ Poncirus trifoliata), e citrumelo 'Swingle' (Citrus paradisi $\mathrm{x}$ Poncirus trifoliata).

A época de coleta dos dados envolveu os anos agrícolas de 1994 a 1998. O controle da produção foi realizado anualmente, computando-se, individualmente, a produção de cada talhão estudado. Os dados sobre produtividade foram obtidos pela divisão da produção colhida (em toneladas) pela área cultivada do talhão (em hectares).

A coleta de folhas, e sua análise, foram realizadas em todos os talhões estudados, em ramos não-frutíferos, nos anos de 1994 a 1998, de acordo com os padrões nutricionais adotados por Hanlon et al. (1995). A coleta das folhas para análise ocorreu sempre nos meses de março ou abril, em ramos com cinco a sete meses de idade.

Os nutrientes analisados foram nitrogênio $(\mathrm{N})$, fósforo $(\mathrm{P})$, potássio $(\mathrm{K})$, cálcio $(\mathrm{Ca})$, magnésio $(\mathrm{Mg})$, enxofre $(\mathrm{S})$, ferro $(\mathrm{Fe})$, manganês $(\mathrm{Mn})$, cobre $(\mathrm{Cu})$, zinco $(\mathrm{Zn})$ e boro (B). As amostras foram enviadas anualmente a laboratórios associados ao Programa de Qualidade, do Instituto Agronômico, Campinas, SP.

As funções das razões dos nutrientes foram calculadas utilizando-se três diferentes recomendações, Beaufils (1973), Jones (1981) e Elwali \& Gascho (1984), descritas a seguir:

Beaufils (1973):

se: $\mathrm{Y} / \mathrm{Xa}<\mathrm{Y} / \mathrm{Xn}$,

então: $f(Y / X)=[1-(Y / X n / Y / X a)] ~ .(100$. k/ CV); se: $\mathrm{Y} / \mathrm{X} \mathrm{Z} \geq \mathrm{Y} / \mathrm{Xn}$,

então: $\mathrm{f}(\mathrm{Y} / \mathrm{X})=[(\mathrm{Y} / \mathrm{Xa} / \mathrm{Y} / \mathrm{Xn})-1] \cdot(100 . \mathrm{k} / \mathrm{CV})$

Beaufils (1973) modificado por Elwali \& Gascho (1984):

se: $\mathrm{Y} / \mathrm{Xa}<\mathrm{Y} / \mathrm{Xn}-\mathrm{s}$,

então: $\mathrm{f}(\mathrm{Y} / \mathrm{X})=[1-(\mathrm{Y} / \mathrm{Xn} / \mathrm{Y} / \mathrm{Xa})] \cdot(100 \cdot \mathrm{k} / \mathrm{CV})$;

se: $\mathrm{Y} / \mathrm{Xn}-\mathrm{s} \leq \mathrm{Y} / \mathrm{Xa} \leq \mathrm{Y} / \mathrm{Xn}+\mathrm{s}$,

então: $\mathrm{f}(\mathrm{Y} / \mathrm{X})=0$;

se: $\mathrm{Y} / \mathrm{Xa}>\mathrm{Y} / \mathrm{Xn}+\mathrm{s}$,

então: $f(Y / X)=[(Y / X a / Y / X n)-1] .(100$. k/CV)

Jones (1981):

$\mathrm{f}(\mathrm{Y} / \mathrm{X})=(\mathrm{Y} / \mathrm{Xa}-\mathrm{Y} / \mathrm{Xn}) \cdot \mathrm{k} / \mathrm{s}$,

em que:

$\mathrm{f}(\mathrm{Y} / \mathrm{X})$ : função calculada da relação de nutrientes $\mathrm{Y}$ e X

$\mathrm{Y} / \mathrm{Xa}$ : relação de nutrientes da amostra;

Y/Xn: relação de nutrientes da norma;

s: desvio-padrão da relação $\mathrm{Y} / \mathrm{Xn}$;

$\mathrm{CV}$ : coeficiente de variação (\%) da relação $\mathrm{Y} / \mathrm{Xn}$;

$\mathrm{k}$ : constante de sensibilidade (neste trabalho, $\mathrm{k}=10$ ).

Dois critérios para a escolha da ordem de relação entre um par de nutrientes A e B foram avaliados. O primeiro critério, denominado de "valor F" (Letzsch, 1985), pressupõe a escolha ou seleção da ordem de relação entre nutrientes pela relação das variâncias $\left(\mathrm{s}^{2}\right)$, da seguinte forma: se:

$\left[\mathrm{s}^{2}(\mathrm{~A} / \mathrm{B})\right.$ pop. referência $/ \mathrm{s}^{2}(\mathrm{~A} / \mathrm{B})$ pop. não-referência $]>\left[\mathrm{s}^{2}(\mathrm{~B} / \mathrm{A})\right.$ pop. referência $/ \mathrm{s}^{2}(\mathrm{~B} / \mathrm{A})$ pop. não-referência],

então: relação da norma $=\mathrm{A} / \mathrm{B}$;

se:

$\left[\mathrm{s}^{2}(\mathrm{~A} / \mathrm{B})\right.$ pop. referência $/ \mathrm{s}^{2}(\mathrm{~A} / \mathrm{B})$ pop. não-referência $]<\left[\mathrm{s}^{2}(\mathrm{~B} / \mathrm{A})\right.$ pop. referência $/ \mathrm{s}^{2}(\mathrm{~B} / \mathrm{A})$ pop. não-referência],

então: relação da norma $=\mathrm{B} / \mathrm{A}$;

em que:

$\mathrm{S}^{2}$ (A/B) pop. referência: variância dos valores $\mathrm{A} / \mathrm{B}$ na população de referência;

$\mathrm{S}^{2}(\mathrm{~A} / \mathrm{B})$ pop. não-referência: variância dos valores de $\mathrm{A} / \mathrm{B}$ na população não-referência;

$\mathrm{s}^{2}(\mathrm{~B} / \mathrm{A})$ pop. referência: variância dos valores $\mathrm{B} / \mathrm{A}$ na população de referência;

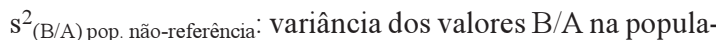
ção de não-referência;

$\mathrm{A} / \mathrm{B}$ e B/A: razão entre os teores de um par de nutrientes $\mathrm{A}$ e B ou, teor de um nutriente $\mathrm{A}$ com base na matéria seca $\mathrm{B}$ (para o M-DRIS).

O segundo critério, denominado de "valor r", proposto e avaliado por Nick (1998), consiste no cálculo dos coeficientes de correlação (r) entre os valores de produtividade e a relação entre os pares de nutrientes, tanto na ordem direta como na ordem inversa. A ordem de relação que resultar no mais alto valor absoluto do coeficiente de correlação (r) é selecionada, conforme é descrito a seguir: 
se: $\left|\mathrm{r}_{\mathrm{A} / \mathrm{B}}\right|>\left|\mathrm{r}_{\mathrm{B} / \mathrm{A}}\right|$,

então: relação da norma $=\mathrm{A} / \mathrm{B}$;

se: $\left|\mathrm{r}_{\mathrm{A} / \mathrm{B}}\right|<\left|\mathrm{r}_{\mathrm{B} / \mathrm{A}}\right|$,

então: relação da norma $=\mathrm{B} / \mathrm{A}$

em que:

$\left|\mathrm{r}_{\mathrm{A} / \mathrm{B}}\right|$ : valor absoluto do coeficiente de correlação entre a produtividade e a razão $\mathrm{A} / \mathrm{B}$ de toda a população (talhões de referência e não-referência);

$\left|\mathrm{r}_{\mathrm{B} / \mathrm{A}}\right|$ : valor absoluto do coeficiente de correlação entre a produtividade e a razão B/A de toda a população (talhões de referência e não-referência);

$\mathrm{A} / \mathrm{B}$ e B/A : razão entre os teores de um par de nutrientes $\mathrm{A}$ e $\mathrm{B}$ ou, teor de um nutriente $\mathrm{A}$ com base na matéria seca $\mathrm{B}$ (para o M-DRIS)

O somatório das funções para cálculo dos índices DRIS foi realizado de acordo com o método M-DRIS (Hallmark et al., 1987). O índice de balanço nutricional (IBN) foi obtido como se segue:

$\mathrm{IBN}=\left|\mathrm{I}_{\mathrm{N}}\right|+\left|\mathrm{I}_{\mathrm{P}}\right|+\left|\mathrm{I}_{\mathrm{K}}\right|+\left|\mathrm{I}_{\mathrm{Ca}}\right|+\left|\mathrm{I}_{\mathrm{Mg}}\right|+\left|\mathrm{I}_{\mathrm{S}}\right|+\left|\mathrm{I}_{\mathrm{Fe}}\right|+\mathrm{I}|\mathrm{Mn}|+\left.\mathrm{I}\right|_{\mathrm{Cu}} \mid$ $+\left.\mathrm{I}\right|_{\mathrm{Zn}}|+\mathrm{I}|_{\mathrm{B}}|+\mathrm{I}|_{\mathrm{MS}} \mid / 12$.

O grupo de dados foi organizado em função dos seguintes fatores: identificação do talhão, variedade porta-enxerto, idade (em anos), número de plantas por hectare. Dados referentes à análise foliar para $\mathrm{N}, \mathrm{P}, \mathrm{K}, \mathrm{Ca}, \mathrm{Mg}, \mathrm{S}, \mathrm{Fe}, \mathrm{Mn}$, $\mathrm{Cu}, \mathrm{Zn}$ e B, de cada um dos talhões, nos anos de 1994 , 1995, 1996, 1997 e 1998, e a respectiva produtividade, foram também organizados

As análises preliminares para teste dos métodos de diagnóstico foram realizadas em populações escolhidas sem discriminação de porta-enxertos, com elevado número de observações. Inicialmente, o método foi avaliado em uma população de talhões de laranjeira 'Valência', com mais de seis anos de idade (pomar adulto em produção estável), enxertada sobre qualquer porta-enxerto (limoeiro 'Cravo', laranjeira 'Caipira', Trifoliata, limoeiro 'Volkameriano', citrange 'Troyer', citrange 'Carrizo', e citrumelo 'Swingle'), isoladamente, nos anos de 1994, 1995, 1996, 1997 e 1998. Foram testadas as combinações de métodos de cálculo das funções de Beaufils, Elwali \& Gascho e Jones e dos valores F e r para a escolha da ordem das razões do índice DRIS, de acordo com os seguintes parâmetros, combinados entre si: produtividade mínima da população de nãoreferência maior ou igual a $20 \mathrm{t} / \mathrm{ha}$ ou $40 \mathrm{t} / \mathrm{ha}$, e produtividade mínima da população de referência maior ou igual a $60 \mathrm{t} / \mathrm{ha}$. Os índices DRIS e IBN foram calculados e os valores de $\mathrm{r}^{2}$ (produtividade em função de IBN) foram calculados para cada método
Em seguida, o mesmo grupo de dados foi novamente analisado. Nessa análise, todos os talhões de laranjeira 'Valência' da Fazenda Sete Lagoas foram organizados e separados em populações, em função de três porta-enxertos (limoeiro 'Cravo', laranjeira 'Caipira', e Trifoliata), os mais freqüentemente utilizados para essa cultivar-copa. A população de talhões foi novamente classificada, em cada porta-enxerto, pela produtividade, para as safras de 1994, 1995, 1996, 1997 e 1998 combinadas em uma análise. Apenas os talhões com mais de seis anos de idade (em produção estável) foram analisados. Os valores de $\mathrm{r}^{2}$ das equações de regressão ajustadas entre os valores de IBN e produtividade foram calculados, com relação aos seis métodos de cálculo DRIS testados, sendo que as populações de não-referência apresentavam produtividade maior ou igual a $40 \mathrm{t} / \mathrm{ha}$, e a população de referência com produtividade maior ou igual a $60 \mathrm{t} / \mathrm{ha}$ em relação ao limoeiro 'Cravo', e maior ou igual a 70 t/ha em relação à laranjeira 'Caipira' e Trifoliata. Esse critério foi adotado devido aos maiores índices de produtividade de laranjeira 'Valência' alcançados sobre esses dois porta-enxertos.

As análises a partir deste ponto, incluíram o detalhamento do agrupamento de dados organizados por ano ou combinação de anos de safra e análise foliar de talhões de laranjeira 'Valência', em função dos três portaenxertos anteriormente estudados, para a definição das normas DRIS para cada combinação enxerto/porta-enxerto. O detalhamento e análise dos dados em anos separados ou em combinações de dois anos foram realizadas para eliminar o efeito das condições do clima na produção.

Finalmente, procedeu-se à seleção criteriosa da população de referência onde foram escolhidos, separadamente para cada combinação copa/porta-enxerto, os talhões de cada grupo que apresentavam grandes diferenças de produtividade em relação aos demais. Conhecendo-se o potencial de produtividade da laranjeira 'Valência' sobre cada porta-enxerto, a separação da população de referência da população não-referência pôde ser realizada com melhor critério. Da população não-referência foram excluídos os talhões que apresentavam boas condições nutricionais, mas baixa produtividade, em razão de outros fatores interferentes na produtividade, tais como, incidência de pragas e doenças. Foram repetidos os passos de determinação das normas e índices DRIS. Registrou-se o valor de $\mathrm{r}^{2}$ da equação ajustada. Com base nesses resultados, dos seis métodos de cálculo DRIS, um foi eleito para melhor representar e calcular os índices DRIS de laranjeira 'Valência' sobre os três porta-enxertos.

\section{Resultados e Discussão}

Os valores de $\mathrm{r}^{2}$ calculados, de um modo geral, são muito baixos, tanto para os critérios de produti- 
vidade mínima de 20 ou de 40 t/ha, como para populações de referência com produtividade mínima de 60 ou $70 \mathrm{t} / \mathrm{ha}$, quando não houve discriminação do porta-enxerto. Uma série de fatores são, então, necessários para o ajuste do método (Tabela 1 ). $\mathrm{O}$ critério de análise escolhido ainda envolve parâmetros muito gerais, principalmente a inclusão de todos os portaenxertos na análise e definições rígidas de população de referência. Em 1998, não foi possível realizar a análise, pois não foram encontrados talhões em número suficiente, com produtividade superior a $70 \mathrm{t} / \mathrm{ha}$ para cálculo dos índices da população de referência. De fato, uma análise mais aprofundada dos índices de produtividade de todos os talhões de laranjeira 'Valência' da Fazenda Sete Lagoas revela que a produtividade decresceu de 1994 a 1998, com aumento do número de talhões com produtividade entre $20 \mathrm{e}$ 40 t/ha e diminuição do número de talhões com produtividade superior a $40 \mathrm{t} / \mathrm{ha}$ (Tabela 2).

No referente aos diferentes métodos de cálculo do DRIS, embora valores muito baixos de $\mathrm{r}^{2}$ tenham sido calculados, mostraram-se relativamente superiores $(0,1230 ; 0,2381$ e 0,2692$)$ os obtidos pelo método Jones (Tabela 1). Quanto ao critério de escolha da ordem da razão dos nutrientes, os resultados não são conclusivos, neste caso.

A população sobre três porta-enxertos (limoeiro 'Cravo', laranjeira 'Caipira' e Trifoliata) apresentou produtividade mínima de 40 t/ha, porque não foram detectadas diferenças nos valores de $\mathrm{r}^{2}$ entre populações de produtividade mínima de 20 ou de 40 t/ha nas análises preliminares (Tabela 1). Também considerou-se que a produtividade da laranjeira 'Valência', em pomares irrigados, quando inferior a 40 t/ha, deve ser atribuída a outros fatores além da nutrição, talvez climáticos, fitossanitários ou relacionados ao manejo. A população de referência escolhida nessa fase apresentou produtividade maior ou igual a $60 \mathrm{t} / \mathrm{ha}$ para limoeiro ‘Cravo' e maior ou igual a 70 t/ha para laranjeira 'Caipira' e Trifoliata, porque a produtividade média sobre aquele porta-enxerto foi inferior aos demais com relação aos anos estudados.

Os valores de $\mathrm{r}^{2}$ das equações de regressão ajustadas entre os valores de IBN e a produtividade de laranjeira 'Valência' (t/ha) sobre os três porta-enxertos escolhidos, para combinações de métodos de cálculo das normas (razões) e índices DRIS (funções),

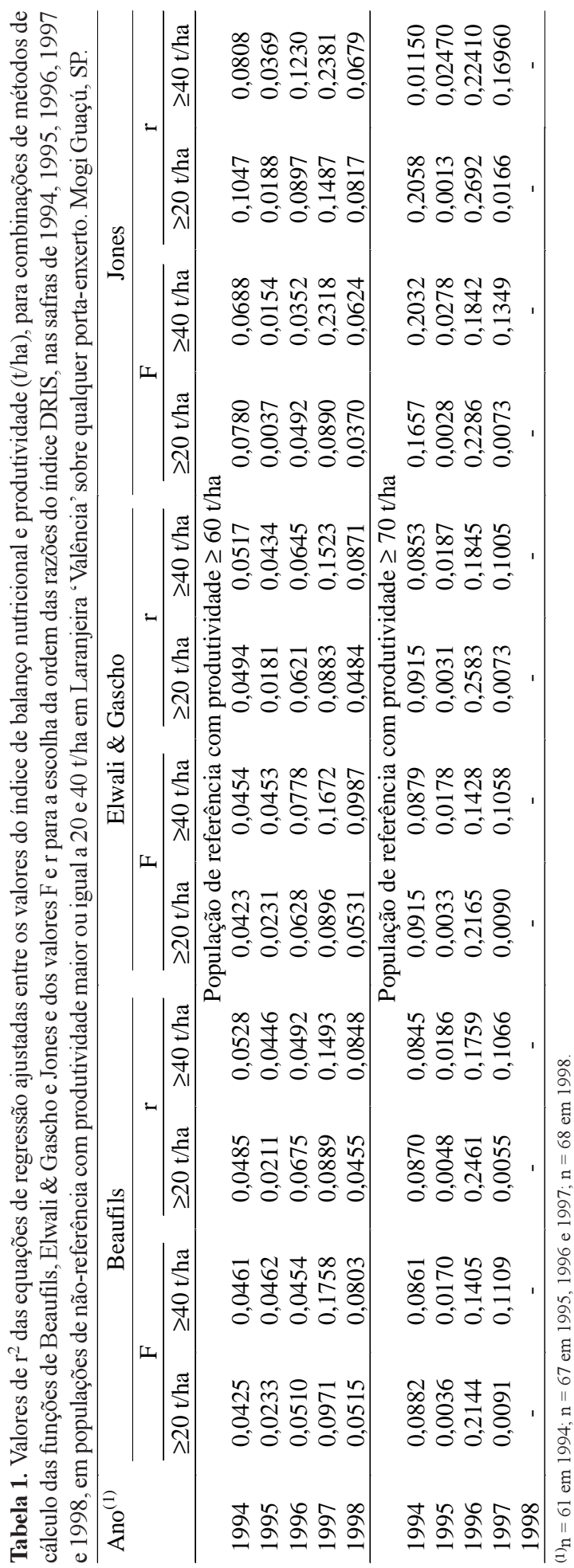

Pesq. agropec. bras., Brasília, v. 37, n. 2, p. 185-192, fev. 2002 
nas safras de 1994 a 1998, em populações de nãoreferência com produtividade maior ou igual a 40 t/ha, encontram-se na Tabela 3. Esses valores calculados ainda são baixos, indicando que outros fatores devem estar mais fortemente relacionados com a variação da produtividade. Entre esses fatores, destacase o fato de muitos anos de safras (1994 a 1998) terem sido incluídos em uma única análise, onde, provavelmente, populações diferentes com padrões nutricionais diferentes, afetadas por pragas e doenças e submetidas a diferentes condições climáticas, apresentaram, ainda, grande variação na produtividade, em virtude de fatores não-nutricionais. Também é de se esperar que, embora um critério mais rigoroso na escolha do tamanho da população tenha sido adotado, excluindo-se os talhões com produtividades inferiores a $40 \mathrm{t} / \mathrm{ha}$, o critério na escolha da população de referência ainda merece ajustes.

Dos métodos de cálculos do DRIS, o preconizado por Jones (1981), valor $r$, produziu maiores valores de $r^{2}$ das equações de regressão ajustadas entre os valores de IBN e produtividade dos porta-enxertos de laranjeira 'Caipira' e Trifoliata, enquanto o de Elwali \& Gascho (1984), valor F, apresentou melhores resultados para o limoeiro 'Cravo' (Tabela 3). Procurou-se, desta forma, prosseguir em análises mais es- pecíficas, na tentativa de isolar todos os fatores nãonutricionais relacionados à produtividade e ajuste da população de referência para cada porta-enxerto.

$\mathrm{Na}$ Tabela 4, encontram-se os valores de $\mathrm{r}^{2}$ das equações de regressão ajustadas entre os valores de IBN e produtividade (t/ha) de laranjeira 'Valência' sobre três porta-enxertos, para combinações de métodos de cálculo das normas (razões) e índices DRIS (funções), em safras selecionadas, em populações de não-referência com produtividade maior ou igual a 40 t/ha e população de referência arbitrada e ajustada caso a caso. Os valores absolutos de $\mathrm{r}^{2}$ das equações de regressão são superiores aos obtidos em análises preliminares, o que indica que as populações utilizadas apresentam variações na produção mais relacionadas a variações na nutrição, variações, essas, relativamente bem avaliadas pelo DRIS.

Quanto às formas de cálculo do DRIS, observase novamente a superioridade do método de somatório das funções recomendado por Jones (1981) em todos os porta-enxertos avaliados, corroborando os resultados obtidos por Santos (1997), em estudos relacionando macronutrientes em experimentos de adubação no Estado de São Paulo. No referente ao critério da escolha da ordem das razões entre os nutrientes, o critério do valor $\mathrm{r}$ acarre-

Tabela 2. Número de talhões de laranjeira 'Valência' sobre qualquer porta-enxerto, com mais de seis anos de idade, com produtividade acima de 20, entre 20 e 40 e acima de 40 t/ha, de 1994 a 1998. Mogi Guaçú, SP.

\begin{tabular}{lccccc}
\hline $\begin{array}{l}\text { Produtividade } \\
\text { (t/ha) }\end{array}$ & 1994 & 1995 & 1996 & 1997 & 1998 \\
\cline { 2 - 5 } & 61 & 67 & 67 & 67 & 68 \\
$>20$ & 6 & 12 & 21 & 28 & 38 \\
$>20<40$ & 55 & 55 & 46 & 39 & 30 \\
\hline 40 & & &
\end{tabular}

Tabela 3. Valores de $\mathrm{r}^{2}$ das equações de regressão ajustadas entre os valores do índice de balanço nutricional e produtividade ( $\mathrm{t} / \mathrm{ha}$ ) para combinações de métodos de cálculo das funções de Beaufills, Elwali \& Gascho e Jones e dos valores (F e r) para a escolha da ordem das razões do índice DRIS, nas safras de 1994 a 1998, em populações de não-referência com produtividade maior ou igual a 40 t/ha de laranjeira 'Valência' sobre três porta-enxertos. População de referência com produtividade maior ou igual a $60 \mathrm{t} / \mathrm{ha}$ para limoeiro 'Cravo' e maior ou igual a 70 t/ha para laranjeira 'Caipira' e Trifoliata. Mogi Guaçú, SP

\begin{tabular}{|c|c|c|c|c|c|c|}
\hline \multirow[t]{2}{*}{ Porta-enxerto $^{(1)}$} & \multicolumn{2}{|c|}{ Beaufils } & \multicolumn{2}{|c|}{ Elwali \& Gascho } & \multicolumn{2}{|c|}{ Jones } \\
\hline & $\mathrm{F}$ & $\mathrm{r}$ & $\mathrm{F}$ & $\mathrm{r}$ & $\mathrm{F}$ & $\mathrm{r}$ \\
\hline Limoeiro 'Cravo' & 0,0268 & 0,0274 & 0,0206 & 0,1920 & 0,0090 & 0,0407 \\
\hline Laranjeira 'Caipira' & 0,0962 & 0,1017 & 0,0811 & 0,0779 & 0,0918 & 0,1276 \\
\hline Trifoliata & 0,1493 & 0,1441 & 0,1511 & 0,1380 & 0,1118 & 0,1578 \\
\hline
\end{tabular}

${ }^{(1)}$ Limoeiro 'Cravo': $\mathrm{n}=52$; laranjeira 'Caipira': $\mathrm{n}=40$; Trifoliata: $\mathrm{n}=77$. 
Tabela 4. Valores de $\mathrm{r}^{2}$ das equações de regressão ajustadas entre os valores do índice de balanço nutricional e produtividade ( $\mathrm{t} / \mathrm{ha}$ ) para combinações de métodos de cálculo das funções de Beaufills, Elwali \& Gascho e Jones e dos valores F e r para a escolha da ordem das razões do índice DRIS, de laranjeira 'Valência' sobre três porta-enxertos, em safras selecionadas, em populações de não-referência com produtividade maior ou igual a 40 t/ha. População de referência arbitrada e ajustada caso a caso. Mogi Guaçú, SP.

\begin{tabular}{|c|c|c|c|c|c|c|}
\hline \multirow[t]{2}{*}{ Porta-enxerto $^{(1)}$} & \multicolumn{2}{|c|}{ Beaufils } & \multicolumn{2}{|c|}{ Elwali \& Gascho } & \multicolumn{2}{|c|}{ Jones } \\
\hline & $\mathrm{F}$ & $\mathrm{r}$ & $\mathrm{F}$ & $\mathrm{r}$ & $\mathrm{F}$ & $\mathrm{r}$ \\
\hline Limoeiro ‘Cravo’ & 0,5801 & 0,6107 & 0,5067 & 0,5511 & 0,6613 & 0,6231 \\
\hline Laranjeira 'Caipira' & 0,5633 & 0,5558 & 0,5537 & 0,5446 & 0,6713 & 0,7358 \\
\hline Trifoliata & 0,3829 & 0,3816 & 0,3887 & 0,3871 & 0,7863 & 0,8275 \\
\hline
\end{tabular}

(1)Limoeiro 'Cravo': n = 12 (safra de 1995, com exclusão dos talhões L204A e L206A); laranjeira 'Caipira': n = 19 (safras de 1994 e 1995 combinadas, com exclusão dos talhões L533A, no ano de 1995 e L404B e L519A no ano de 1995); Trifoliata: $\mathrm{n}=16$ (safra de 1994).

tou valores de $r^{2}$ das equações de regressão mais elevados que o critério do valor $\mathrm{F}$ para os porta-enxertos laranjeira 'Caipira' e Trifoliata (Tabela 4), o que concorda com as pesquisas de Nick (1998) em cafeeiros podados. Para o limoeiro 'Cravo', o método F apresentou $r^{2}$ pouco superior ao calculado pelo método $\mathrm{r}(0,6613$ contra 0,6231 , respectivamente) (Tabela 4).

Fatores determinantes que contribuíram para a obtenção de valores mais elevados de $\mathrm{r}^{2}$ das equações de regressão foram a separação da população por porta-enxerto e por safra, ou combinação de safras, e escolha criteriosa da população de referência, ou seja, escolha do grupo de plantas ou talhões mais representativos do que seria o padrão ou o máximo em relação à produtividade, quando todos os fatores não-nutricionais foram isolados.

Em especial, o fato de a população de laranjeira 'Valência' ter sido selecionada em uma região de cultivo irrigado e classificada quanto ao porta-enxerto beneficiou o isolamento desses fatores. Rodriguez et al. (1997), na definição de normas DRIS com relação à laranjeira 'Valência', na Venezuela, não encontraram diferenças ou influência dos porta-enxertos limoeiro ‘Volkameriano' e tangerina 'Cleópatra'. Entretanto, a influência do porta-enxerto na composição mineral das folhas de laranjeira 'Valência' já é conhecida e foi pesquisada (Wutscher, 1982).

Os métodos de Beaufils (1973) e de Elwali \& Gascho (1984) não apresentaram diferenças quanto aos valores de $r^{2}$ das equações de regressão, concordando com os resultados de Bataglia \& Santos (1990). O método Jones (1981) foi superior aos de- mais, também concordando com o obtido por Nick (1998) para cafeeiro (Tabela 4).

Em decorrência do critério de o valor r para escolha da ordem das razões dos nutrientes ter sido superior para os porta-enxertos limoeiro 'Cravo' e laranjeira 'Caipira', adotou-se Jones (1981), pelo valor $\mathrm{r}$ (Nick, 1998) como o melhor método de cálculo do DRIS em laranjeira 'Valência'.

\section{Conclusões}

1. A separação da população por porta-enxerto e por safra ou combinação de safras, juntamente com a escolha criteriosa da população de referência são fatores determinantes na obtenção de valores mais elevados de $r^{2}$ nas equações de regressão entre índices DRIS e produtividade.

2. Populações específicas, com pequeno número de observações, padronizadas quanto ao porta-enxerto e referentes a um ou dois anos de amostragem foliar e produção são bancos de dados eficientes para a obtenção das normas DRIS.

3. O método de cálculo do DRIS, com base no somatório das funções recomendado por Jones, apresenta melhor correlação com produtividade do que os métodos cujos cálculos baseiam-se em Beaufils e em Elwali \& Gascho, principalmente para a laranjeira 'Caipira' e Trifoliata, sendo com menor intensidade para o limoeiro 'Cravo'.

4. O critério do valor $r$ mostra-se mais eficiente que o valor $\mathrm{F}$ para a escolha da ordem da razão dos nutrientes para a laranjeira 'Caipira' e Trifoliata, sendo que o valor $\mathrm{F}$ é mais eficiente que o $\mathrm{r}$ para o limoeiro 'Cravo'. 


\section{Agradecimentos}

Ao Grupo Sete Lagoas Agrícola S/A, pelo apoio concedido para a realização deste trabalho.

\section{Referências}

BATAGLIA, O. C. DRIS-Citros: uma alternativa para avaliar a nutrição das plantas. Laranja, Cordeirópolis, v. 10 , p. $565-576,1989$

BATAGLIA, O. C.; SANTOS, W. R. dos. Efeito do procedimento de cálculo e da população de referência nos índices do sistema integrado de diagnose e recomendação (DRIS). Revista Brasileira de Ciência do Solo, Campinas, v. 14, p. 339-344, 1990.

BEAUFILS, E. R. Diagnosis and recommendation integrated system (DRIS). Soil Science Bulletin, Natal, n. 1, p. $1-132,1973$

BEVERLY, R. B. A practical guide to the diagnosis and recommendation integrated system (DRIS). Athens: Micro-Macro, 1991. 87 p.

BEVERLY, R. B. Prescient diagnostic-analysis shows sufficiency range approach superior to DRIS for citrus Communications in Soil Science and Plant Analysis, New York, v. 23, p. 2641-2649, 1992.

CERDA, A.; NIEVES, M.; MARTINEZ, V. An evaluation of mineral analysis of Verna lemons by DRIS Communications in Soil Science and Plant Analysis, New York, v. 26, p. 1697-1707, 1995.

CRESTE, J. E. Uso do DRIS na avaliação do estado nutricional do limoeiro siciliano. Botucatu: Unesp, 1996. 120 p. Tese de Doutorado.

ELWALI, A. M. O.; GASCHO, G. J. Soil testing, foliar analysis, and DRIS as guide for sugarcane fertilization Agronomy Journal, Madison, v. 76, p. 466-470, 1984.

HALLMARK, W. B.; MOOY, C. J. de; PESEK, J. Comparison of two DRIS methods for diagnosing nutrient deficiencies. Journal of Fertilizer Issues, Manchester, v. 4, p. 151-158, 1987.

HANLON, E. A.; OBREZA, T. A.; ALVA, A. K. Tissue and soil analysis In: TUCKER, D. P. H.; ALVA, A. K.; JACKSON, L. K.; WHEATON, T. A. Nutrition of Florida citrus trees. Gainesville: University of Florida, 1995. p. 13-16
JONES, W. W. Proposed modifications of the diagnosis and recommendation integrated system (DRIS) for interpreting plant analyses. Communications in Soil Science and Plant Analysis, New York, v. 12, p. 785 794, 1981.

LETZSCH, W. S. Computer program for selection of norms for use in the diagnosis and recommendation integrated system (DRIS). Communications in Soil Science and Plant Analysis, New York, v. 16, p. 339347,1985

LETZSCH, W. S.; SUMNER, M. E. Effect of population size and yield level in selection of diagnosis and recommendation integrated system (DRIS) norms. Communications in Soil Science and Plant Analysis, New York, v. 15, p. 997-1006, 1984.

MORENO, J. J.; LUCENA, J. J.; CARPENA, O. Effect of the iron supply on the nutrition of different citrus variety/rootstock combinations using DRIS. Journal of Plant Nutrition, New York, v. 19, p. 689-704, 1996

NICK, J. A. DRIS para cafeeiros podados. Piracicaba: Esalq, 1998. 86 p. Dissertação de Mestrado.

RODRIGUEZ, O.; ROJAS, E.; SUMNER, M. Valencia orange DRIS norms for Venezuela. Communications in Soil Science and Plant Analysis, New York, v. 28 , p. $1461-1468,1997$

SANTOS, W. R. dos. Avaliação do equilíbrio nutricional dos macronutrientes em citros com diferentes adubações. Piracicaba: Esalq, 1997. 112 p. Dissertação de Mestrado.

WALLACE, A. Nitrogen, phosphorous, potassium interaction on Valencia orange yields. Journal of Plant Nutrition, New York, v. 13, p. 357-365, 1990.

WOODS, D. B.; VILLIERS, J. M. Diagnosing the nutrient status of 'Valencia' oranges in Southern Africa. In: INTERNATIONAL CITRUS CONGRESS, 7., 1992, Acireale. Proceedings... Acireale: International Society of Citriculture, 1992. p. 556-559

WUTSCHER, H. K. The influence of medium heterogeneity and three rootstocks on growth and nutrient levels of greenhouse-grown 'Valencia' orange trees. Journal of the American Society for Horticultural Science, Alexandria, v. 107, p. 235-239, 1982 